INPLASY

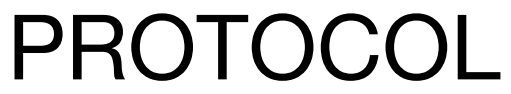

To cite: Moridi et al. CABG versus myotomy in symptomatic myocardial bridge patients: A systematic Review and Meta-analysis protocol. Inplasy protocol 2021110088. doi: 10.37766/inplasy2021.11.0088

Received: 24 November 2021

Published: 24 November 2021

Corresponding author: Parinaz Onikzeh

parinaz_onikzeh@yahoo.com

Author Affiliation:

Shahid Beheshti University of Medical Sciences, Tehran, Iran.

Support: Self funded.

Review Stage at time of this submission: Preliminary searches.

Conflicts of interest:

None declared.

\section{CABG versus myotomy in symptomatic myocardial bridge patients: A systematic Review and Meta-analysis protocol}

Moridi, M1; Onikzeh, P2; Kazemi, $\mathrm{A}^{3}$; Zamanian, $\mathrm{H}^{4}$.

Review question / Objective: The aim of this study is to find which surgical intervention in myocardial bridge ( myotomy or CABG) is more effective in reducing adverse outcomes in symptomatic patients resistant to optimal medical therapy? Condition being studied: Myocardial bridge : A myocardial bridge (MB) is a congenital heart defect in which a bridge of muscle fibers (myocardium) overlying a section of a coronary artery and the artery is squeezed and normal blood flow is disrupted. Most bridges don't seem to cause symptoms. However, some people can experience angina, or chest pain. In patients with symptoms, first line treatment is medication and if they have symptoms despite optimal medical treatment, invasive measures like CABG or myotomy should be taken.

INPLASY registration number: This protocol was registered with the International Platform of Registered Systematic Review and Meta-Analysis Protocols (INPLASY) on 24 November 2021 and was last updated on 24 November 2021 (registration number INPLASY2021110088). 
is squeezed and normal blood flow is disrupted. Most bridges don't seem to cause symptoms. However, some people can experience angina, or chest pain. In patients with symptoms, first line treatment is medication and if they have symptoms despite optimal medical treatment, invasive measures like CABG or myotomy should be taken.

\section{METHODS}

Search strategy: PUBMED search strategy ("Miocardial bridge" OR "Miocardial bridging" OR "myocardial bridge" OR "myocardial bridging" OR "muscle bridge" OR "muscle bridging" OR "bridge" OR "bridging" OR "intramiocardial bridging" OR "intramyocardial bridging" OR "intram y ocardial bridge" OR "intramiocardial bridge" OR "myocarial milking" OR "miocardial milking" OR "intramiocardial milking" OR "intramyocardial milking" OR "functional obstruction" OR "LAD obstruction" OR "LAD functional obstruction" OR "left anterior descending functional obstruction" OR "left anterior descending obstruction" OR "anterior interventricular branch obstruction" OR "anterior interventricular branch functional obstruction" OR"anterior descending branch functional obstruction" OR "coronary artery functional obstruction" OR "coronary artery obstruction" OR "left coronary artery obstruction" OR "left coronary artery functional obstruction" OR "coronary blood vessel functional obstruction" OR "coronary blood vessel obstruction" OR "left anterior descending" OR "arteries functional obstruction" OR "functional obstruction of LAD" OR "functional obstruction of left anterior descending" OR "functional obstruction of anterior interventricular branch of left coronary artery" OR "functional obstruction of anterior descending" OR "functional obstruction of coronary" OR "descending artery bridge" OR "LAD bridge" OR "coronary artery bridge" OR "coronary artery obstruction" OR "anterior descending branch bridge" OR "anterior descending branch obstruction") ("CABG" OR "Coronary Artery Bypass" OR "Cardiopulmonary Bypass" OR "Cardiac
Surgical Procedures" OR "MIDCAB" OR "MIDCABG" OR "aortocoronary bypass" OR "aortocoronary vein" OR "saphenous bypass" OR "saphenus bypass" OR "aortocoronary saphenous" OR "saphenous vein graft" OR "graft" OR "bypass surgery" OR "cardiac surgery" OR "thoracic surgery" OR "bypass" OR "open heart surgery" OR "heart surgery" OR "internal mammary-coronary artery anastomosis") ("myotomy" OR "myectomy" OR "unroofing" OR "unroof").

Participant or population: Myocardial bridge patients confirmed by Angiography or CT-Angiography who are resistant to optimal medical therapy and are candidate for CABG or Myotomy, have not received any invasive treatment previously.No age and sex limitation in participants will be considered.

Intervention: On/off pump myotomy which involves resecting the overlying muscle fibers of the myocardial bridge through median sternotomy.

Comparator: On/off pump CABG (coronary artery bypass graft surgery) which using LIMA (left internal mammary artery) through median sternotomy.

Study designs to be included: Randomised control trials, non-randomised clinical trials, Case series,

Eligibility criteria: The articles will be included only if they are original articles on hospitalized myocardial bridge patients who are candidate for CABG or myotomy procedure and should have both myotomy intervention and CABG intervention groups and have comparison between the outcome of these groups .Patients should not have received any invasive treatment before.without any restrictions in age or gender in participants.

Information sources: Systematic literature search in digital databases: Scopus, Web of Science, PubMed, Embase, Manual searches of reference lists applied to identify any studies not found in the electronic searches. 
Main outcome(s): -Major adverse cardiac events (MACE) which is defined as the composite of total death, myocardial infarction, coronary revascularization, stroke, and hospitalization -Recurrent angina -Complete relief of pain.

Additional outcome(s): All-cause mortality after intervention.

Data management: Data will be extracted from databases and will be imported to RAYYAN and duplicated articles will be removed. Titles and abstracts of studies will be screened by two reviewers independently and relevant studies will be selected. Then we will screen the full texts of selected articles to include appropriate articles by considering inclusion and exclusion criteria. In all above steps when confronting any disagreement, the third reviewer will help to resolve uncertainty. data extraction will be performed using a pre-designed data extraction form considering the following items of each included article: article title, first author information, year of publication, study aim, location of the study group, type of study, participant characteristics( total sample size, sample drop out, age, number of myotomy and CABG cases, etc), localization, extension, and depth of myocardial bridge, treatment of choice ( CABG vs myotomy), report of surgery side effects, follow up test or scan, information relevant totrial design, the percent of MACE(major adverse cardiac events) including death, myocardial infarction, revascularization, re hospitalization, recurrence of chest pain in each surgery group, percent of repeat re vascularization, CCS class, composite adverse events in each surgical group, residual compression of tunneled artery percent in myotomy group , LIMA graft patency rate in CABG group two members of team will extract data in an excel sheet independently from finally included articles and the third member will check these data.

Quality assessment / Risk of bias analysis: Modified jadad scale.
Strategy of data synthesis: We will conduct a systematic review synthesis and we will use text and tables to explain our findings for qualitative synthesis and if it will be possible to perform meta-analysis based on studies outcomes, we will do metaanalysis using CMA and STATA Softwares.

Subgroup analysis: No planned subgroups.

Sensitivity analysis: Not applicable in this systematic review.

Country(ies) involved: Iran.

Keywords: myocardial bridge, CABG, Myotomy, Angina.

Contributions of each author:

Author 1 - Mina Moridi.

Email: mina_m292002@yahoo.com

Author 2 - Parinaz Onikzeh.

Email: arinaz_onikzeh@yahoo.com

Author 3 - Aida Kazemi.

Email: aidakzm1991@gmail.com

Author 4 - Hadi Zamanian.

Email: hadi_zamanian@yahoo.com 